\title{
Plano de Controle GMPLS para Redes Ópticas de Transporte
}

\author{
F.P.Favoreto, A.S.Garcia, M.T.A.Torres, R.S.Tessinari e M. E. V. Segatto
}

\begin{abstract}
Resumo-Neste trabalho é apresentada uma proposta de implementação do plano de controle GMPLS para redes OTN. As normas do ITU-T e IETF relativas às duas tecnologias foram relacionadas a fim de garantir uma proposta genérica e consistente, resolvendo questões que não são abordadas nas recomendações do GMPLS relativas a redes OTN. É proposta também a modelagem de um sistema de simulação que seja capaz de tratar esta abordagem, garantindo que o sistema como um todo esteja funcionando de acordo com recomendações vigentes.
\end{abstract}

Palavras-Chave-GMPLS, OTN, protocolos de sinalização, redes ópticas, plano de controle.

Abstract-In this work a GMPLS control plane implementation applied to OTN networks is presented. The ITU-T and IETF recommendations related to both technologies were taken into consideration, so we could get a general and consistent proposition, which can deal with issues not treated in the GMPLS recommendations about OTN. We also propose the modeling of a simulating system able to deal with this view, so we are able to know if the system is working as the recommendations state they should.

Keywords-GMPLS, OTN, signaling protocols, optical networks, control plane.

\section{INTRODUÇÃO}

O crescimento contínuo do tráfego da Internet demanda a cada momento uma nova tecnologia para transportar os dados a velocidades cada vez maiores. O surgimento de outras fontes de tráfego, como dispositivos móveis, com diferentes requerimentos de QoS, constituem um desafio cada vez maior para a convergência de redes. Neste contexto as tecnologias de redes ópticas de transporte surgiram como uma alternativa para o núcleo das redes dada a sua alta taxa de transmissão e confiabilidade.

As redes OTN (Optical Transport Network), baseadas na multiplexação DWDM (Dense Wavelength-Division Multiplexing) e nas já existentes SONET/SDH (Synchronous Optical NETwork/Synchronous Digital Hierarchy), surgiram como uma tecnologia capaz de responder a esse desafio.

Inicialmente essas redes eram compostas por dispositivos com pouca flexibilidade como OADM's (Optical Add/Drop Multiplexers), onde a topologia e todo o processo de WA (Wavelength Assignment) são feitos em tempo de projeto da rede. Recentemente os ROADM's (Reconfigurable Optical

Felipe Pedroni Favoreto, Anilton Salles Garcia, Marcos Tadeu de Araújo Torres, Rodrigo Stange Tessinari e Marcelo E. V. Segatto, Programa de Pós Graduação em Engenharia Elétrica, Universidade Federal do Espírito Santo, Vitória, Brasil, E-mails: feliperama@gmail.com, anilton@inf.ufes.br, ermactadeu@gmail.com, rodrigostange@gmail.com, segatto@ele.ufes.br. Este trabalho foi parcialmente financiado pela PADTEC S.A.
Add/Drop Multiplexers) agregaram maior flexibilidade à rede, além possibilitar a aplicação de métodos de engenharia de tráfego, assím como o uso de mecanismos de proteção e restauração.

No entanto, o crescimento em complexidade das redes OTN com o uso de dispositivos reconfiguráveis implica em custos de operação cada vez mais altos. O GMPLS surge como uma solução de plano de controle a fim de permitir o provimento de serviços de forma transparente sobre a rede OTN.

Este trabalho tem como objetivo relacionar as recomendações do ITU sobre redes OTN e as recomendações do IETF relativas ao GMPLS a fim de obter uma solução consistente e genérica relativa ao plano de controle. Embora o framework GMPLS especificado pelo IETF possa atuar em redes com comutação de comprimentos de onda, ainda existem questões a serem resolvidas, como, por exemplo, representar os recursos de uma rede OTN, ou aproveitar a gerência de falhas indicada na recomendação ITU-T G.798 [1] no gerenciamento de links do GMPLS.

Verificou-se na literatura atual que esse tipo de abordagem ainda não foi explorada. Em [2] os autores tratam da integração do plano de controle GMPLS (Generalized Multi Protocol Label Switching) e a rede WDM através de uma arquitetura UNI (User Network Interface). Em [3] são propostas extensões para o protocolo OSPF (Open Shortest Path First) de modo que ele seja capaz de tratar questões de engenharia de tráfego mais eficientemente no GMPLS. O trabalho [4] propõe um esquema dinâmico para controle de conexões que satisfaz os novos requerimentos do GMPLS e onde a rede de controle está separada da rede de transporte. Em [5] é demonstrado em um testbed como o plano de controle proposto seria capaz de garantir o aprovisionamento de circuitos ópticos de forma automatizada. Todos esses trabalhos descrevem soluções baseadas no GMPLS para redes ópticas de maneira geral, sem considerar detalhes específicos da tecnologia OTN, como a equivalência entre componentes arquiteturais ou a correlação de defeitos nativa.

Na próxima seção é descrita a base teórica utilizada neste artigo, os principais conceitos de OTN e GMPLS necessários ao entendimento do artigo. Na seção III é discutido o uso do GMPLS em uma rede OTN. Na seção IV é apresentada a proposta de implementação e simulação das soluções sugeridas na seção III, enquanto as conclusões e sugestões de trabalhos futuros estão na seção V.

\section{TECNOLOGIAS ENVOLVIDAS}

Nesta seção são apresentados os principais aspectos das tecnologias OTN e GMPLS e das recomendações que as 
definem, relacionados com a proposta apresentada.

\section{A. OTN}

De acordo com [6], OTN é uma tecnologia que foi desenvolvida para ser uma evolução das redes de transporte de núcleo, combinando a tecnologia DWDM, que permite a transmissão de múltiplos comprimentos de onda em uma única fibra óptica, com os benefícios da tecnologia SONET/SDH. Uma rede OTN é composta por um conjunto de ONE's (Optical Network Element), interconectados por links de fibras ópticas, e capazes de prover as seguintes funcionalidades: transporte, multiplexação, roteamento, gerenciamento, supervisão e mecanismos de sobrevivência para os canais ópticos, contendo os sinais cliente.

A recomendação ITU-T G.805 [7] define funcionalmente uma série de elementos e modela uma rede genérica de transporte. Baseada nessas definições a recomendação ITUT G.872 [8] descreve a arquitetura de uma rede OTN e, a partir dessa, em ITU-T G.709 [9] são definidas as interfaces, as funcionalidades do cabeçalho e a estrutura dos frames. É na recomendação ITU-T G.798 [1] que uma descrição funcional da hierarquia OTN é detalhada e divida em duas partes: a Hierarquia Óptica de Transporte (HOT) e Hierarquia Digital de Transporte (HDT). Cada hierarquia é subdividida em camadas, com funcionalidade principal bem definida no contexto OTN.

A HDT é composta por: OPU (Optical Channel Payload Unit) que realiza o tratamento do sinal digital cliente, efetuando operações de justificação quando necessário; a ODU (Optical Channel Data Unit) que realiza a multiplexação digital, fornecendo um caminho digital fim a fim ao sinal cliente; e a OTU (Optical Channel Transport Unit), que fornece um caminho digital entre dois NEs e FEC (Forward Error Correction). Cada OTU é mapeada diretamente em um canal óptico.

A HOT tem como componentes: o OCh (Optical Channel Layer), a primeira camada óptica, onde acontecem as conversões digital/óptico e óptico/digital. Provê um caminho óptico fim a fim, iniciando e finalizando um comprimento de onda; a OMS (Optical Multiplex Section), camada responsável por multiplexar os comprimentos de onda originados das camadas OCh em um único sinal óptico; e a OTS (Optical Transmission Section) que fornece um caminho óptico entre dois ONE's. Na OTS o sinal óptico originado na camada OMS é multiplexado com o sinal de cabeçalho, gerando o sinal que é transmitido na fibra.

OTN baseia-se no conceito de camada servidora e camada cliente, onde cada camada oferece algum serviço para a camada imediatamente acima. Por exemplo, a camada OCh é a camada servidora da camada OTU, por oferecer um caminho óptico para a transmissão dos dados digitais. A mesma camada OCh é cliente da camada OMS. De forma similar a uma rede baseada no modelo de referência OSI (ou TCP/IP), cada camada adiciona um cabeçalho ao payload e envia para a camada inferior através da terminação de trilha da camada servidora [7]. Assim, o payload de uma camada servidora é, na verdade, o payload da camada cliente com o respectivo cabeçalho da mesma. No entanto, nas camadas ópticas (OCh,
OMS, OTS) o payload é adicionado apenas no canal de supervisão.

\section{B. GMPLS}

As Redes Ópticas, apesar da grande largura de banda, acabam apresentando problemas semelhantes às redes baseadas em pacotes quando se pensa na necessidade de gerenciá-las. O GMPLS engloba um conjunto de protocolos do plano de controle, e provê suporte para controlar diferentes tecnologias de tráfego de transporte e serviço.

O GMPLS pode ser visto como uma extensão da arquitetura MPLS permitindo o interfaceamento com tecnologias que não são apenas baseadas na comutação de pacotes, estendendo o paradigma label switching para tecnologias orientadas à conexão, provendo suporte para conexões TDM (Time Division Multiplexing), incluindo SONET, SDH e OTN. Ou seja, o GMPLS inclui os dispositivos capazes de realizar: (a) Comutação de pacotes; (b) Multiplexação por divisão de tempo; (c) Comutação de lambda (ou comprimento de onda); (d) Comutação waveband; (e) Comutação de fibras.

Os protocolos do GMPLS podem ser agrupados em dois planos distintos: $\mathrm{O}$ plano de roteamento e o plano de sinalização, juntos chamados de plano de controle. Os protocolos de sinalização do GMPLS são capazes de criar, manter, modificar e terminar caminhos de dados, que no contexto do (G)MPLS são chamados de LSP (Label Switched Path). Os protocolos de roteamento no GMPLS não cuidam do "roteamento"propriamente dito, mas da distribuição da informação a ser usada como base no cálculo dos LSP's a serem alocados na rede.

Os dispositivos que comutam os dados baseando-se apenas nos rótulos (e portas) de chegada são denominados LSRs (Label Switched Routers). Os LSR são gerenciados por controladores GMPLS que implementam o plano de sinalização e roteamento. Normalmente esses controladores ficam fisicamente dentro dos LSR's, embora possa estar fisicamente separado controlando mais de um elemento da rede de transporte. Isso permite a existência de LSR ópticos, capazes de comutar os dados diretamente na camada óptica sem necessidade de interpretar no nível elétrico os dados transmitidos. Por exemplo, um controlador no plano de controle pode gerenciar um OXC (Optical Cross-Connect) do plano de transporte, dizendo a ele como rotear os dados que chegam.

\section{USO DO GMPLS EM REDES OTN}

As recomendações IETF relativas ao GMPLS apresentam um conjunto de protocolos de sinalização, roteamento e descoberta a fim de adaptar as funcionalidades do MPLS para redes não-pacotes TDM, LSC (Lambda Switch Capable), FSC (Fiber Switch Capable). A maior parte dessas recomendações trata de forma genérica o plano de transporte. Neste sentido, o principal desafio é identificar como garantir a usabilidade dos protocolos associados ao GMPLS com a arquitetura OTN.

$\mathrm{Na}$ arquitetura OTN existem dois tipos de multiplexação: 1)a digital, na camada ODU, e 2)a óptica, na camada OMS [8]. Devido a isso, a arquitetura OTN permite o estabelecimento de dois tipos de LSP's, um TDM no nível digital e outro 
LSC no nível óptico. Os limites de um LSP da camada digital correspondem, no plano de transporte, ao início e fim de uma trilha ODU, enquanto os limites de um LSP da camada óptica são o início e fim de uma trilha OCh.

\section{A. Informações para o plano de roteamento}

Para escolher o caminho a ser utilizado por um LSP, o protocolo de roteamento do plano de controle precisa representar as informações sobre os recursos do plano de transporte, que são guardadas no banco de dados de engenharia de tráfego TED (Traffic Engineering Database). O TED é usado por protocolos de roteamento como o OSPF-TE [12] ou por um PCE (Path Computation Element) [17] e tem como função guardar, através de uma estrutura de grafos, todas as informações necessárias sobre as entidades de transporte em um domínio. O principal componente do TED é o TE link (Traffic Engineering Link), o qual representa um agrupamento de recursos para fins de roteamento. O propósito de representar diversos recursos físicos em apenas um recurso lógico é de diminuir a quantidade de TE links no TED. Para representar a OTN no TED é necessário identificar em uma OTN quem são os nós do grafo e quais são os TE links. A figura 1 ilustra a representação desses recursos de uma rede OTN.

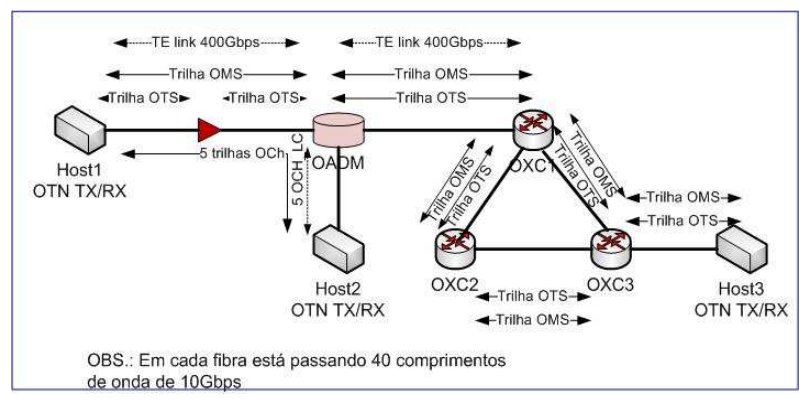

Fig. 1. Exemplo de uma OTN e as terminações de trilha.

O amplificador de linha é um dispositivo de terminação de trilha apenas da camada OTS. Na representação do TED ele pode ser transparente, pois a sua existência em princípio não interfere na escolha de uma rota. Sendo assim, ele não é um ponto de terminação de um TE link.

O TED deve conter também as informações de restrições de roteamento. No exemplo da figura 1, suponha que se deseja alocar um LSP do Host2 ao Host3, passando pelos nós OADM, OXC1, OXC3, Host3. Se o OXC1 não possuir conversão de comprimento de onda, então o comprimento de onda alocado entre o OXC1 e o OXC3 precisa ser o mesmo comprimento de onda alocado entre o OADM e o OXC1. Essas informações de restrições são relativas aos nós do grafo e são retiradas das restrições da matriz de conexão da função de conexão da camada OCh.

\section{B. Informações para o plano de Sinalização}

$\mathrm{Na}$ sinalização GMPLS as informações relativas aos recursos da OTN estão na tabela de rótulos existente em cada LSR. Segundo [13], um rótulo quando relacionado a um plano de transporte TDM, LSC ou FSC representa um recurso físico da rede, que está sendo utilizado por um LSP. É importante notar que o rótulo sempre representa o recurso mínimo a ser alocado. Para um LSP no domínio óptico, um rótulo representa um dos comprimentos de onda multiplexados na camada OMS, enquanto que em um LSP no domínio digital o rótulo representa um dos ODUk componentes (um timeslot) multiplexados na camada da camada ODU.

Quando um rótulo de entrada está disponível na tabela de rótulos significa que o comprimento de onda que ele representa não foi reservado ainda para o LSR de upstream, portanto tem-se um comprimento de onda disponível. Quando um rótulo de saída está disponível significa que se tem um comprimento de onda disponível para uso com o LSR de downstream. Neste contexto a tabela de rótulos precisa obter do plano de transporte informações sobre quantas são as portas (as fibras com um sinal WDM no padrão OTN) de entrada e saída, qual a capacidade dessas (quantos e quais são os comprimentos de onda) e quais as restrições quanto à alocação desses comprimentos de onda, para garantir a correta representatividade da rede OTN. A parte de restrições funciona exatamente como no TED.

\section{Endereçamento e Representação dos TE links}

Em [13] é introduzido o conceito de agrupamento de links, onde um TE link, em vez de representar diretamente um canal físico de uma rede, representa um conjunto de links componentes. Para isso é necessário que: 1) as terminações de cada link componente pertençam ao mesmo par de LSR's e 2) possuam o mesmo tipo de link, métrica ou custo administrativo. No caso de uma OTN, o primeiro agrupamento que deve ser considerado é dos comprimentos de onda de uma mesma trilha OMS. Se, além disso, existem várias fibras entre dois elementos ópticos (ROADM, OXC, OADM), então se pode também agrupar as fibras e criar apenas um único TE link que represente a capacidade de troca de informações entre dois dispositivos.

Tipicamente a identificação de um recurso da rede é feita utilizando a dupla <identificador do TE link, rótulo>. Porém, em alguns casos de agrupamento essa quantidade de informação não é suficiente para identificar de forma unívoca um recurso. Segundo [14] são necessários três componentes: $<$ identificador do TE link (agrupado), identificador do link componente, rótulo $>$. Considere o exemplo de várias fibras entre dois OXC's transportando sinais WDM, onde o plano de controle representa tudo em um único TE link. Neste caso o identificador do link componente seleciona uma das fibras dentro do agrupamento de fibras, enquanto o rótulo identifica o comprimento de onda utilizado.

Os identificadores, tanto do TE link quanto do link componente, podem ser numerados ou não-numerados. Numerado significa que o identificador é um endereço IP, e não numerado pode ser qualquer outro tipo de identificador. No caso de endereços não-numerados os dois LSR's de terminação de um link devem trocar entre si esses identificadores, o que pode ser feito via configuração, por um protocolo como o LMP (Link Management Protocol), pelo RSVP-TE (Resource ReServation 
Protocol - Traffic Engineering)/CR-LDP (Constraint-Routing Label Distribution Protocol) ou por extensões do protocolo de roteamento IS-IS (Intermediate System to Intermediate System) ou OSPF.

\section{Gerenciamento de Links da OTN}

Além de identificar os recursos na rede transporte, o plano de controle precisa gerenciar o estado desses recursos, a fim de manter sua base de dados sempre atualizada caso haja alguma falha de um componente do plano de transporte. O IETF criou o LMP [15] que, dentre outras funções, é responsável pelo gerenciamento de falhas através de troca de mensagens pelo canal de transporte quando ele não está sendo utilizado. Isso exige que em cada terminação do canal seja feita uma conversão digital para que o LMP possa processar as mensagens, o que não ocorre nas terminações das camadas ópticas da OTN. Esse processo se faz desnecessário uma vez que uma OTN possui seus próprios mecanismos de verificação de falhas, que podem ser aproveitados pelo plano de controle.

Em uma OTN as terminações de trilha são responsáveis por realizar uma série de verificações, tanto da carga útil quanto no cabeçalho. Na recomendação ITU-T G.798 [1] é apresentado como funciona a verificação de falhas em cada uma das camadas da arquitetura OTN. Em linhas gerais, nas camadas da parte óptica (OTS, OMS e OCh) sinais de erro são gerados com base na observação através de foto-detector dos sinais ópticos, tanto da carga útil quanto do canal de controle. Em seguida é feita a correlação de defeitos e os resultados são disponibilizados para o plano de gerência ou de controle. Nas camadas digitais o processo é o mesmo, porém o sinal observado é uma PDU e não um sinal óptico, portanto, em vez de foto-detector, são observados erros de bit na carga útil ou no cabeçalho.

Quando há uma falha de perda de sinal na trilha OTS ou OMS, todos os comprimentos de onda transportados por essas trilhas são desativados. Essa informação deve ser passada automaticamente para o plano de controle para que ele possa atualizar sua base de dados.

\section{Proposta de ImplementaÇÃo e Simulação NO OMNET++}

Nesta seção é apresentada a modelagem de simulação para o mapeamento proposto na seção 3 , baseada na arquitetura GMPLS para o plano de controle, a fim de demonstrar como é possível realizar o estabelecimento de LSP's ópticos da camada OCh da OTN. A simulação relativa à parte digital do mapeamento, correspondente ao tratamento dos LSP's da camada ODU, está em fase de construção.

\section{A. Proposta de Implementação}

Uma das principais diferenças entre o GMPLS e o MPLS está na separação do plano de controle do plano de transporte. O controlador é o componente arquitetural responsável por um ou mais elementos do plano de transporte [18]. Os controladores se comunicam através de uma DCN (Data Communications Network), uma rede logicamente separada da rede de dados (a que transporta o sinal cliente), mas que pode fisicamente compartilhar recursos com a rede de transporte. Para simplificar o desenvolvimento da simulação, cada elemento da OTN que faz terminação de um TE link tem o seu próprio controlador. Na figura 2 tem-se como exemplo dois LSRs adjacentes, o primeiro formado pelo Controlador1 e o OXC1, e o segundo formado pelo Controlador2 e o OXC2.

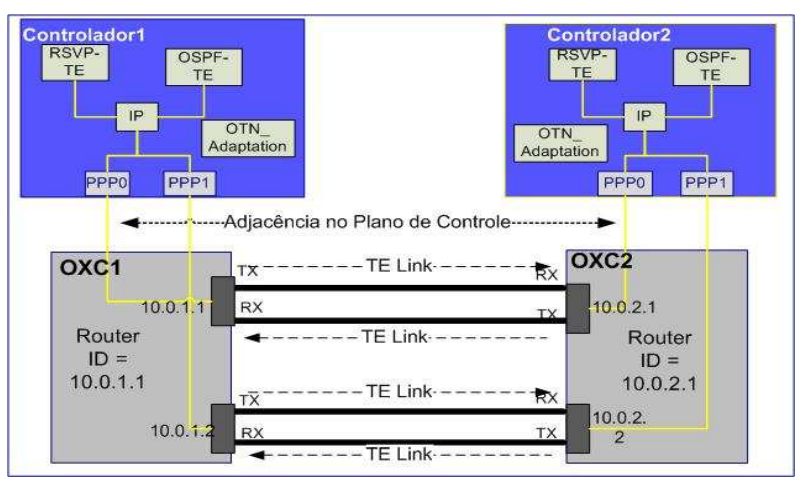

Fig. 2. Comunicação entre dois LSRs adjacentes.

A agregação de links em um TE link é composta por todas as conexões de link da camada OCh que fazem parte da mesma trilha OMS, ou seja, todos os comprimentos de onda da fibra com exceção do OSC (Optical Supervisor Channel). Os valores da taxa de transmissão de cada comprimento de onda devem ser somados no TE link. No caso de uma conexão bidirecional, correspondente a um par de fibras entre dois OXC's, existem duas trilhas OMS, uma em cada direção. Esse par de fibras é representado na TED por dois TE links. Para identificar unicamente dentro do TE link um comprimento de onda, usa-se a tupla <identificador do TE link, rótulo $>$, onde o identificador do TE link corresponde ao endereço IP associado aquela fibra e o rótulo representa o comprimento de onda a ser utilizado. Por exemplo, na figura 2 se o Controlador1 deseja configurar o comprimento de onda zero na primeira fibra para transmitir dados, ele irá se referir a este recurso através da tupla $\langle 10.0 .1 .1,0\rangle$, considerando que zero representa o primeiro comprimento de onda em uma fibra.

$\mathrm{Na}$ arquitetura OTN o canal de supervisão, ou OSC, é o comprimento de onda pelo qual são enviados os cabeçalhos das camadas OTS, OMS e OCh. Este sinal é multiplexado juntamente com todos os outros comprimentos de onda na camada OTS e o seu formato específico é deixado para estudos futuros pelas normas do ITU-T para OTN [1], permitindo que o fabricante defina qual será o tipo de tecnologia a ser utilizada. Por outro lado, na RFC3945 [13] não se faz nenhuma especificação quanto a tecnologia do canal de controle, embora deixe claro a preferência por tecnologias do mundo IP. Uma forma de convergir as soluções é utilizar o OSC para o transporte de informações de cabeçalho OTN e de mensagens dos protocolos do GMPLS. Para o plano de controle, o fato de que o sinal está sendo transportado pelo OSC é completamente transparente. 


\section{B. Simulação}

A simulação para uso do plano de controle GMPLS em equipamentos OTN é desenvolvida no simulador OMNeT++ [19]. São implementados no simulador: um canal óptico, que representa uma fibra com sinais WDM e atenuação de potência, a implementação do plano de transporte OTN e um controlador GMPLS.

No OMNeT++ não existe um canal óptico para implementação, sendo necessária, então, a criação de um novo tipo de canal. O canal WDM construído no modelo de simulação permite que sejam transmitidos diversos quadros OTN de forma simultânea e independente, desde que em comprimentos de onda diferentes.

A implementação dos módulos OTN é baseada na recomendação ITU-T G.798[1], onde são implementadas as camadas ópticas OTS, OMS e OCh. Cada equipamento OTN, como, por exemplo, um OXC ou um Amplificador de Linha, são simulados utilizando-se os blocos funcionais de terminação de trilha e de adaptação descritos na recomendação G.798, onde cada bloco funcional é modelado por um módulo específico no OMNeT++. A figura3 a seguir ilustra um exemplo da modelagem desenvolvida, neste caso um OXC, que implementa funcionalmente as camadas OTS e OMS.

Um equipamento OTN é composto de um conjunto de elementos físicos como (de)multiplexadores, amplificadores ópticos, fotodetectores entre outros. No entanto, os módulos criados tomando-se por base a recomendação G.798 são modelos funcionais, que ao serem conectados e configurados corretamente modelam qualquer equipamento OTN. Por exemplo, um OXC do mundo real tem um estágio de pré-amplificação se existirem amplificadores ópticos em cada fibra de entrada. Ao invés disso, esse estágio é configurado no simulador através de um parâmetro no sub-módulo OTSn_OMSn_A_Sk do OXC, que aciona essa funcionalidade.

A simulação do plano de controle GMPLS é implementada através da extensão dos módulos já existentes para MPLS na API INET [20]. Na figura 3 apenas o sub-módulo OTN_ADAPTATION do GMPLSControler não existia e foi implementado neste módulo. Basicamente, a OTN_Adaptation possui informações atualizadas do $\mathrm{OXC}$, que são: 1)uma entrada para cada fibra com o endereço IP associado, 2) informações com os alarmes gerados pelas terminações de trilha OTN e funções que calculam o estado do link, ativo ou com problemas, de acordo com esse alarme, 3) função para a configuração de rotas do OXC.

GTED (Generalized Traffic Engineering Database) é a implementação da tabela de engenharia de tráfego e foi modificada para que cada TE link representado em uma estrutura de grafos contenha a capacidade de transmissão de todos os comprimentos de onda somados. Para isso, a GTED troca informações com a OTN_Adaptation sobre as fibras que estão conectadas ao OXC, bem como sua capacidade de transporte.

O módulo GRSVP (Generalized ReSerVation Protocol), responsável pela sinalização, é a extensão do módulo RSVPTE existente no INET de acordo com RFC3471[10], 3473[11] e 4328[16] do IETF, a fim de que ele possa ser usado para estabelecer LSP's no domínio óptico. As principais mudanças da sinalização foram realizadas na mensagem de

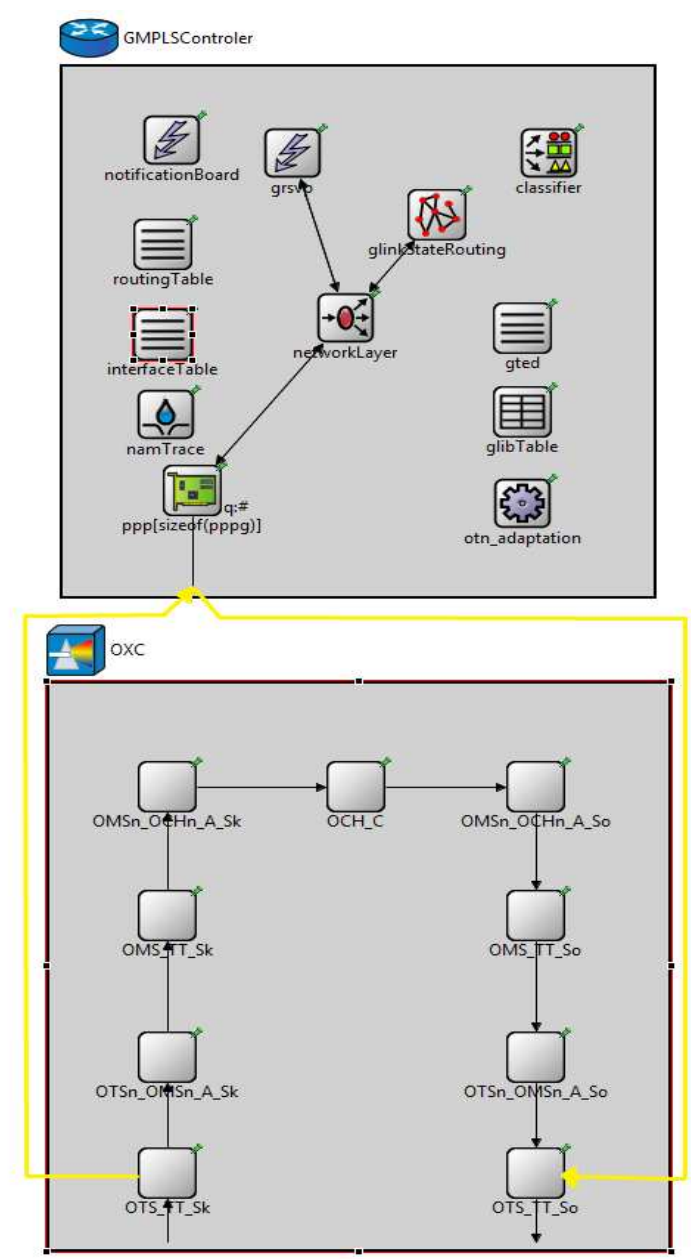

Fig. 3. Simulação do LSR, formado por um OXC aclopado a um controlador GMPLS.

PATH, responsável pela criação do caminho óptico. O ERO (Explicit Route Object) contém, parcial ou integralmente, o caminho que deverá ser usado no estabelecimento do LSP através dos sub-objetos LABEL ERO [10] qual deve ser o comprimento de onda utilizado entre cada par de OXCs . O objeto GENERALIZED LABEL_REQUEST, que possui informações gerais sobre o tipo de LSP a ser estabelecido, inclui os campos LSP Encoding Type, Switching Type e GPID [10], preenchidos de acordo com [16] a fim de indicar uma LSP no nível óptico. Foram adicionados na mensagem de PATH os objetos SUGGESTED_LABEL, o qual possui uma lista de rótulos (comprimentos de onda) que podem ser usados no sentido downstream, e UPSTREAM_LABEL, que indica qual será o comprimento usado no sentido UPSTREAM, no caso do estabelecimento de conexões bidirecionais [10]. Esses campos representam os comprimentos de onda utilizados entre dois LSRs do caminho óptico, e é alterado a cada nó de acordo com os LABELs contidos no ERO.

O objeto SENDER_TSPEC transporta os parâmetros de tráfego que detalham as informações sobre a LSP. Esse objeto foi alterado para incluir os parâmetros ST (Signal Type), 
NMC (Number of Multiplexed Components), NVC (Number of Virtual Componentes) e MT (MulTiplier), os quais são específicos da tecnologia OTN. Por exemplo, ST=7 indica que está sendo requisitado para a LSP um OCh de $10 \mathrm{Gbps}, \mathrm{NVC}=0$ indica que não há concatenação virtual e $\mathrm{MT}=1$ significa que apenas uma instância desse sinal deverá ser transmitida. NMC deve ser sempre 0 no estabelecimento de LSPs no nível óptico [16].

Quando a mensagem de PATH alcança o seu destino, no LSR de saída da rede, o ultimo LSR envia de volta uma mensagem de RESV para confirmar a alocação dos comprimentos de onda no caminho óptico. O objeto FLOWSPEC é idêntico ao objeto SENDER_TSPEC. Na figura 4 tem-se um exemplo do uso do GMPLS para a alocação de rotas. Os controladores em cada LSR enviam as mensagens de PATH para criar caminhos ópticos na rede em malha. Os HOSTs representam clientes da rede OTN e geram um sinal óptico OTN completo, onde o HOST1 troca dados com o HOST3, e o HOST2 troca dados com o HOST4.

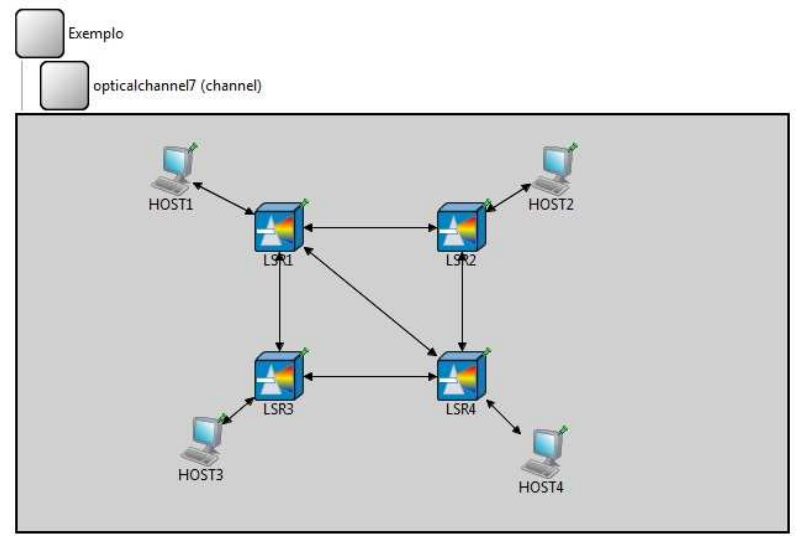

Fig. 4. Exemplo de rede OTN com LSR's com plano de controle GMPLS.

\section{Conclusões}

Este trabalho apresenta uma análise aprofundada da relação entre as normas ITU-T sobre redes OTN e das RFCs do IETF sobre GMPLS. Este estudo tem como prioridade analisar os componentes arquiteturais das redes OTN especificados pela ITU-T e mostrar como estes recursos devem ser representados e manipulados pelo plano de controle GMPLS. Através dessa análise são sugeridas algumas mudanças nos processos de gerenciamento de links a fim de aproveitar os mecanismos de detecção e correlação de falhas providas pela OTN, evitando assim a sobreposição de funcionalidades.

O uso do GMPLS em redes OTN é demonstrado através de um protótipo desenvolvido no simulador de eventos discretos OMNeT++. Módulos de simulação de redes OTN foram criados com base na norma ITU-T G798, incluindo um canal óptico WDM. A simulação do plano de controle GMPLS é feita através da extensão dos módulos para MPLS com base nas recomendações do IETF RFC3945, RFC3473, RFC3471 e RFC4328.

Assim, o artigo demonstra como utilizar o plano de controle GMPLS sobre redes OTN, relacionando as recomendações do
ITU e IETF e através de modelos de simulação. O trabalho contribui no sentido de comparar os modelos utilizados pelos dois órgãos, além de apresentar quais são as análises que devem ser realizadas para aplicar os protocolos do GMPLS em uma tecnologia de transporte específica.

Para trabalhos futuros sugere-se a implementação das camadas digitais da OTN, a extensão do plano de controle para a alocação de LSP's na camada ODU, o estabelecimento de H-LSP's (Hierarchical LSP's) e a extensão do TED e dos protocolos de roteamento para o uso de mecanismos de RWA (Routing Wavelenght Assignment).

\section{REFERÊNCIAS}

[1] ITU-T, Telecommunication Standardization Sector of ITU. G.798. Characteristics of optical transport network hierarchy equipment functional blocks. 14 de Dezembro de 2006.

[2] R.Pasquini,F.L.Verdi,L.G.Zuliani,M.Magalhaes,S.Rossi, "An Optical UNI Architecture for the GIGA Project Testbed Network," Telecommunications Symposium, 2006 International(ITS'06), pp.285-290, Set. 2006.

[3] L. G. Zuliani, G. S. Pavani, M. Savasini, R. Pasquini, F. L. Verdi e M. Magalhães, "An implementation of an OSPF-TE to support GMPLS-controlled all-optical WDM networks," VI IEEE International Telecommunications Symposium (ITS'06), pp.300-305, Setembro 2006.

[4] Y.Hyeonsik e K.Kwangjoon, "Dynamic Connection Control Scheme Considering Network Topology in an Optical Network," Advanced Communication Technology, 2008. ICACT 2008. 10th International, Vol. 3, pp.1680-1683, Fev. 2008.

[5] F.D.Santos, G.C.Santos, e J.C.R.F.OLIVEIRA, "Aprovisionamento Automático de Circuitos Ópticos via Plano de Controle GMPLS aplicado a uma Rede Óptica Reconfigurável Baseada em ROADMs," XXVI Simpósio Brasileiro de Telecomunicações, 2008.

[6] Iniewski, Krzysztof, McCrosky, Carl e Minoli, Daniel., Network Infrastructure And Architecture. New Jersey : Wiley Interscience, 2008.

[7] ITU-T, Telecommunication Standardization Sector of ITU. G.805. Generic functional architecture of transport networks. 10 de Março de 2000.

[8] ITU-T, Telecommunication Standardization Sector of ITU. G.872. Architecture of optical transport networks. 29 de Novembro de 2001.

[9] ITU-T, Telecommunication Standardization Sector of ITU. G.709. Interfaces for the Optical Transport Network (OTN). 16 de Março de 2003.

[10] L. Berger, Generalized Multi-Protocol Label Switching (GMPLS) Signaling Functional Description, RFC3471, IETF, Jan. 2003.

[11] L. Berger, Generalized Multi-Protocol Label Switching (GMPLS) Signaling Resource ReserVation Protocol-Traffic Engineering (RSVP-TE) Extensions, RFC3473, IETF, Jan. 2003.

[12] D. Katz, K. Kompella, D. Yeung, Traffic Engineering (TE) Extensions to OSPF Version 2, RFC3630, IETF, Set. 2003.

[13] E. Mannie, Generalized Multi-Protocol Label Switching (GMPLS) Architecture, RFC3945, IETF, Out. 2004.

[14] K. Kompella, Y. Rekhter, L. Berger, Link Bundling in MPLS Traffic Engineering (TE), RFC4201, IETF, Out. 2005.

[15] J. Lang, Link Management Protocol (LMP), RFC4204, IETF, Out. 2005.

[16] D. Papadimitriou, Generalized Multi-Protocol Label Switching (GMPLS) Signaling Extensions for G.709 Optical Transport Networks Control, RFC4328, IETF, Jan. 2006.

[17] A. Farrel, J.-P. Vasseur, J. Ash, A Path Computation Element (PCE)Based Architecture, RFC4655, IETF, Ago. 2006.

[18] A.Farrel, I.Bryskin., GMPLS: Architecture and Applications. s.l. : Morgan Kaufmann Publishers, 2006.

[19] A.Varga, "The Omnet++ Discrete Event Simulation System," Proceedings of the European Simulation Multiconference, Jun. 2001.

[20] INET Framework for OMNeT++ 4.0 . [Online] [Citado em: 20 de 05 de 2009.] http://inet.omnetpp.org/.

[21] R.S.Tessinari, Mapeamento de Equipamentos Ópticos e Modelagem de Redes OTN no Simulador OMNeT++ de acordo com a Recomendação ITU-T G.798, Departamento de Informática, Engenharia de Computação, Universidade Federal do Espírito Santo, Brasil, Jan.2009. 